\title{
Comparative Evaluation of Neuropathological Lesions Progression in Different Brain Areas in A Rat Model of Chronic Hepatic Encephalopathy
}

\author{
Eltahir M. Mohamed ${ }^{1}$, Faten F. Mohammed ${ }^{2 *}$, Hala El Miniawy ${ }^{2}$ \\ ${ }^{1}$ College of veterinary Medicine, Sudan University of Science and \\ Technology, Sudan, (Postgraduate student, Department of Pathology, \\ Faculty of veterinary medicine, Cairo University) \\ ${ }^{2}$ Department of Pathology, Faculty of veterinary medicine, Cairo \\ University, Giza, 12211, Egypt. \\ *Corresponding author: fatenfathy21@yahoo.com,ORCID : \\ https://orcid.org/0000-0003-0410-
}

\begin{abstract}
Hepatic encephalopathy (HE) is considered as serious clinical complication of acute and chronic liver injury. Previous researches elucidated $\mathrm{HE}$ but characterization of neuropathology in different brain areas is limited.The present study is conducted to evaluate the progression of neuropathological lesions in different brain areas in rat model with liver fibrosis induced by thioacetamide (TAA). For this experiment 20 male rats was divided into two groups control and TAA treated group that was I.P injected twice a week with $100 \mathrm{mg} / \mathrm{kg} . \mathrm{b} . \mathrm{w}$ TAA dissolved in saline for 3 months. Three rats from each group were euthanized after 3 months and the liver specimens were collected for ensuring the development of rat model for liver fibrosis. The remained rats were kept without further treatment with TAA for 2 months, rats were carfeully monitored for abnormal signs. After the end of experimental period, rats were euthanized and the brains were collected for histopathological examination. Microscopic examination of brain revealed various neurpoathological lesions that varied in severity between different brain areas. The most severe lesions were recorded in midbrain and pons while mild lesions were detected in cerebral cortex and striatum. The brain lesions comprised varied degrees of neuronal degeneration with marked astroglial and microglial reactions in the different brain regions. The obvious inflammatory reaction indicates that the neuroinflammation play an important role in the mediation of HE.
\end{abstract}


Key words: Cirrhosis, hepatic encephalopathy, rats.

\section{Introduction}

Hepatic encephalopathy (HE) is a complex neuropsychiatric clinical condition developed in patients with liver diseases, which may develop to several neurological and psychiatric alterations that affect cognition and lead to motor impairment and can progress to coma and death (Weissenborn et al., 2004). HE developed as a complication of acute and chronic liver failure (Farjam et al, 2012)

The neurological manifestations developed with acute and chronic liver injury were defined into: type A represents HE with acute hepatic failure (ALF), type $B$ is rare and was defined to be the neuropsychiatric complication of portal-systemic by pass without any intrinsic hepatocellular pathology and type $\mathrm{C}$ is the involvement of the brain seen in cirrhotic patients (Weissenborn et al., 2004, Monto liu et al., 2010, and Butterworth, 2011).

Thioacetamide (TAA) is used widely for the induction of experimental liver fibrosis (Wallace et al., 2015) ,on other hand TAA-induced hepatic encephalopathy model is one of the most popular one for acute hepatic disorders (Butterworth et al., 2009 and Bismuth et al., 2011).

The pathogenesis of $\mathrm{HE}$ developed in liver disease is related to hyperammonemia that associated with acute and chronic liver injury (Cauli et al., 2014). In addition, neuroinflammation rather than brain edema are implicated in the pathogenesis of HE (Rodrigo et al., 2010, and Butterworth, 2011).

The present work was conducted to perform intensive study on chronic hepatic encephalopathy associated with chronic liver disease through microscopic examination of neuropathological lesions progression in different brain areas in thioacetamid treated rats.

Material and methods

Animals and experimental design

Experiments were performed on adult male Wistar rats, weighting 120-150 g, that were purchased from VACSERA. Animals were kept in clean metal cages with pelleted food and tap water ad libitum. All experimental procedures were approved by the Ethical Committee of the Faculty of veterinary medicine, Cairo 
University (Vet.CU.IACUC-VetCU03252019026-25/3/2019).

All animals $(\mathrm{n}=20)$ were divided into two groups 10 rats each, group A kept as control untreated group ,and group B was I.P injected with TAA (Lobachemei-India code no.0625900100) at dose of 100 $\mathrm{mg} / \mathrm{kg}$ B.w two times weekly for 3 months (Dongmei Qin, et al.,2014). Three rats from each group were euthanized after 3 months from the beginning of experiment and liver specimens were collected to check the development of liver fibrosis model.

The remained rats were kept without further treatment for 2 months. Rats were euthanized at the end of the experimental period, and the brain tissues were collected for histopathology.

Histopathogical examination

Brain specimens were collected in $10 \%$ buffered formalin, routinely processed, and stained according to Bancroft (2013). Tissue sections were examined using Olympus BX43 light microscope and captured using Olympus DP27 camera linked to Cellsens dimensions software (Olympus) with microscopic examination different brain areas including cerebral cortex, striatum, hippocampus, midbrain, cerebellum and pons.

Results

Clinical signs
The treated rats lost the normal behavior, like grooming and continuous movement in the cage. The rats were sluggish, showed incoordination and slowly moved in the cage compared with control one.

Histopathological alterations

The microscopic examination of different brain areas revealed variuos histopathological alterations that vary in nature and severity among different brain areas.

\section{1-Cerebral cortex}

The lesions in cerebral cortex were mild in severity and restricted to cerebral grey matter. It included endothelial capillary proliferation (Fig.1a) and hypertrophy, in addition to distenstion of perivascular area especially in the inner granular and pyramidal layers.

\section{2-Striatum}

The lesions in striatum were similar to that detected in cerebral cortex. It involved mostly the caudate ,putamen and the globus pallidus with mild microgliosis (rod cells) (Fig.1b) with no reactions were detected in internal capsule.

\section{3-Hippocampus}

There was cellular reduction of the pyramidal neuronal cells involving all layers of cornus ammonis (CA). The lesion characterized by loss of neurons with gliosis and hypertrophy of endothelium. The cellular reduction was more severe in 
CA3 \&CA4 (Fig.2a). The dentate hilus showed gliosis and neuronal degeneration of pyramidal neurons. In individual case, there was a focal atsrocytosis associated with neuronal loss. (Fig.2b).At the periphary of such lesion, there was reactive astrocytes appearred enlarged with abundant eosinophilic cytoplasm (Fig.2c).on the other hand individual Alzehiemer type II astrocytes were detcted in the hippocampus hilus.

\section{4-Thalamus}

It showed different degree of neuronal degeneration varied from central chromatolysis associated with gliosis (Fig.3a) to severe lesion charcterized by massive loss of neurons with vacuolation of neuropil associated with reactive astrocytosis and microgliosis (Fig.3b). There was also perivascular mononuclear cells aggreagtion $(\mathrm{Fg} .3 \mathrm{c})$.

\section{5-Cerebellum}

Concerning cerebellum,the lesions were more severe comapred with cerebrum. The lesions included microgliosis of molecular layer,focal loss of purkinje cells (Fig.4a) the cerebellar white matter showed microgliosis and endothelail capillary proliferation (Fig.4b). Vacuolation of neuropil with microgliosis and chromatolysis of large neuronal cells were also seen in the cerebellar dentate nucleus(Figs. 4c).

\section{6-Midbrain and Pons}

The lesion in mid brain and pons were more severe comapred with other brain areas. It involved the reticulate part of substantia nigra and characterized by neuronal necrosis and microgliosis (Figs.5a\&b).

The lesions in pons charcterized by chromatolysis and necrosis of large neurons of pontine nuclei assocaited with intense glial reaction microgliosis and astrocytosis (Fig.5c).The glia reaction represented reactive microglia (rod cells) and reactive astrocytes( Gemistocytes) admixed with other histicytes (Fig.5d).The lesion was also associted with hypertrophy of capillary endothelium. 


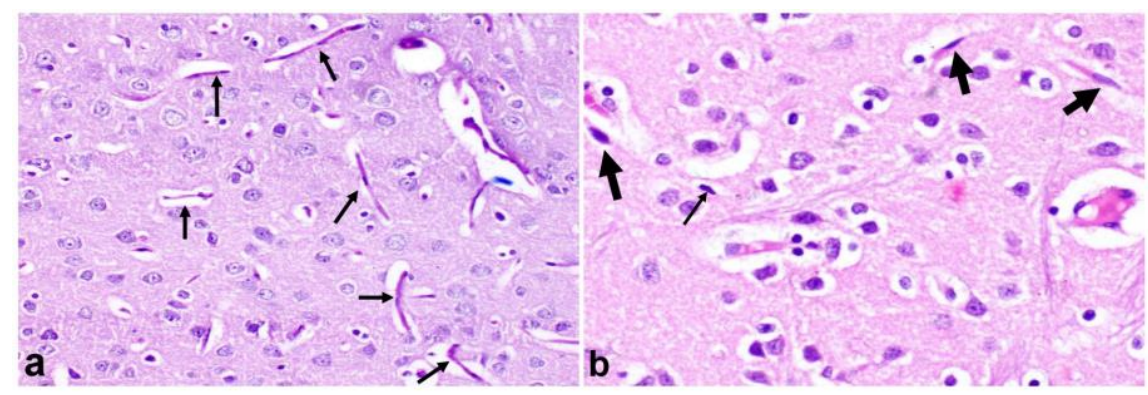

Fig.1: a) Cerebrum of rat model showing endothelial capillary proliferation (arrow) X 200. b) Caudate showing Endothelial lining hypertrophy (thick arrow), note the presence of rod cell (thin arrow) X400.

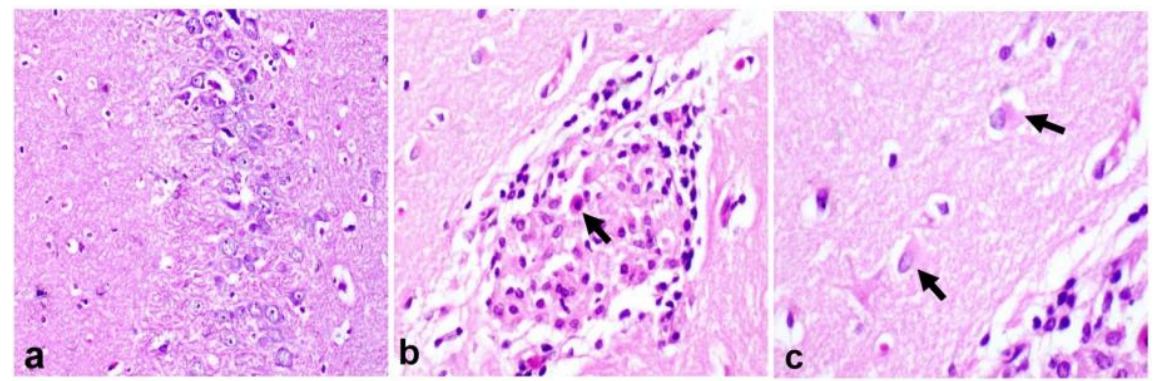

Fig.2: Hippocampus of rat model showing: a) massive cellular reduction and loss of large pyramidal neurons in CA4 layer X200. b) focal necrosis of pyramidal neurons (arrow) in the dentate hilus invaded with microglia and astrocytes X400. c) Higher magnification of previous image, note the reactive astrocytes(arrow) X600.

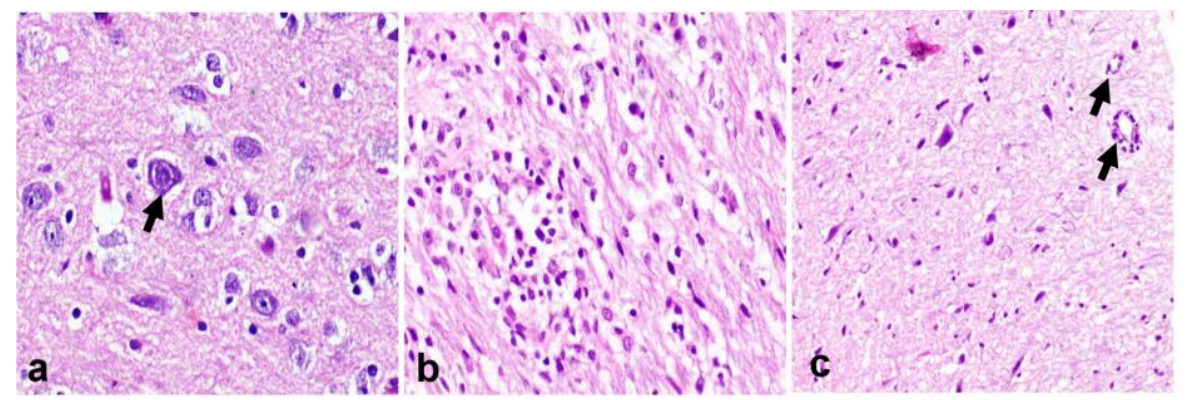

Fig.3: Thalamus of rat model showing a) central chromatolysis of pyramidal neurons (arrow) with gliosis X400. b) Encephalomalcia with microgliosis and reactive astrocytosis X400. c) Perivascular mononuclear cell aggregation (arrow) X200. 


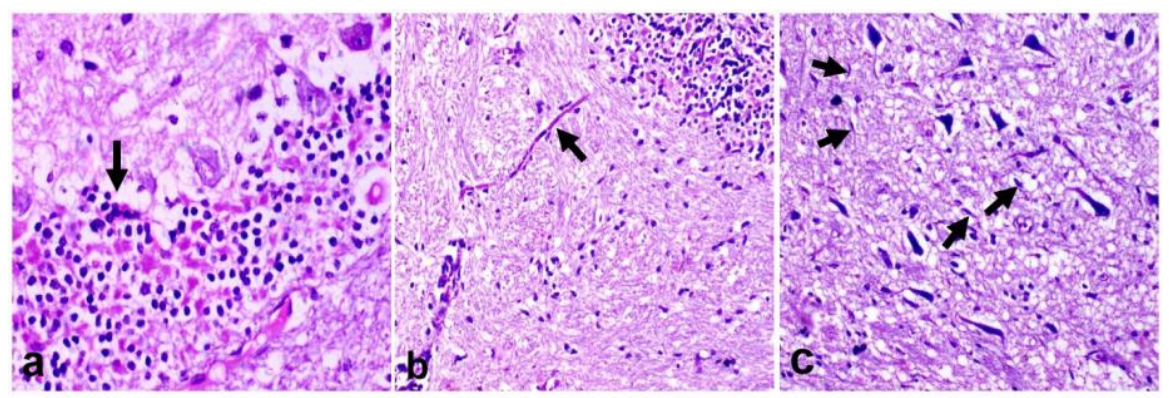

Fig.4: Cerebellum of rat model showing: a) Focal loss of Purkinje cells (arrow) associated with microgliosis of molecular layer X400. b) Microgliosis of the cerebellar white matter with endothelial capillary proliferation (arrow) and perivascular mononuclear cells aggregation X200 c) cerebellar dentate nucleus showing diffuse gliosis mainly microglia (arrow) with vacuolization of neuropil X20O.

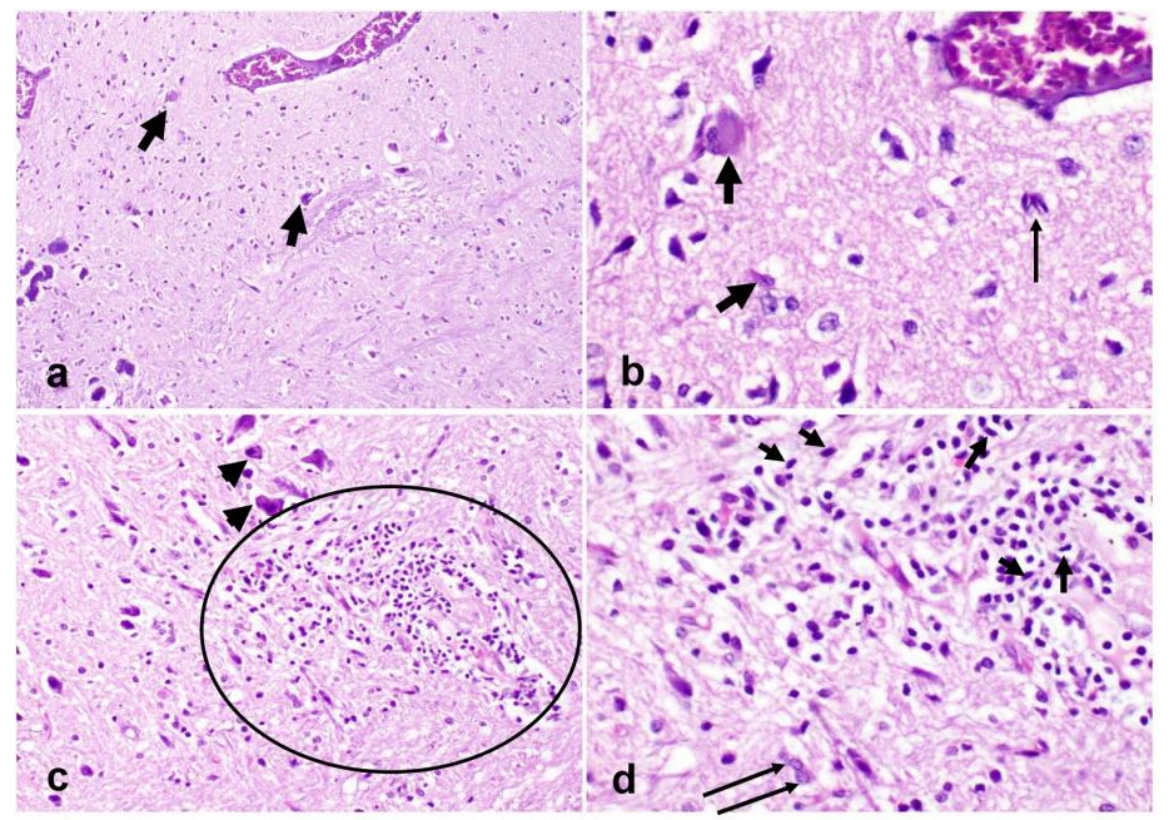

Fig.5: Mid brain and pons of rat model showing a) necrosis of neurons (arrow) comprising the substatia nigra reticularis X100. b) Necrosis of large nerve cells (thick arrow) associated with microgliosis (thin arrow) X400. c) Pons with chromatolysis and necrosis of neurons comprising the pontine nuclei associated with intense glial reaction (circle) X200. d) Higher magnification of the previous image, note the microgliosis with typical rod cells (short arrow) admixed with other histiocytes and reactive astrocytes (long arrow) X400. 


\section{Discussion}

The present work revealed that $\mathrm{HE}$ is associated with progression of neuropathological lesions comprising neuronal injury and cellular reaction that vary in severity among different brain areas. Concerning the cellular reaction in the brain of rat model, astrocytosis were detected in areas of neuronal necrosis or associated with foci of microgliosis, while Alzheimer type 2 astrocytes were detected incidentally in dentate hilus of hippocampus. There was a correlation between the presence of Alzheimer type 2 astrocytes in HE and increase ammonia level in blood as discussed by Butterworth (2009) who reported that HE in rat model developed Alzheimer type 2 astrocytosis and he added that one of pathophysiology of $\mathrm{HE}$ is due to increased blood ammonia. On contrary Cauli et al. (2013) found low blood ammonia level in rats with chronic liver disease and develop HE. The microgliosis was extensively seen in areas of hippocampus, thalamus, midbrain, cerebellum and pons not in cerebral cortex. These results indicate the neuroinflammation mediated injury in these areas with subsequent development of clinical signs. Butterworth (2011) stated that microgliosis was developed in $\mathrm{HE}$ in cirrhosis and indicative of proinflammatory mechanism. Previous researches stated that the neuroinflammation and ammonia toxicity were considered as one of predominant factor in developing $\mathrm{HE}$ in acute and chronic liver diseases (Rodrigo et al., 2010, Butterworth, 2011, and Cauli et al., 2014).

Neuronal injury, degeneration and loss were seen in the brain but the lesions were varying in severity according to brain areas. The most severe neuronal degeneration and loss were detected in pons and mid brain areas and associated with intense reactive astrogliosis and microgliosis.

Butterworth (2009) stated that deep cerebral cortex, basal ganglia and cerebellum developed spongiform degeneration associated with hepatic cirrhosis, on contrary we found that the neuronal loss and degeneration were scarce in basal ganglia, cerebral cortex and cerebellum.

The neuronal loss and neuroinflammation reflected on behavior of rats that showing slow motion and loss of abnormal behavior of arts like grooming and exploring behavior in spite of normal feeding habits. Previous researches were conducted on progression of nervous signs 
with HE into stages, mild associated with mild cognition deficits and cannot be detected by general examination (Huaussinger et al., 2006, and Romero-G et al., 2001) and moderate includes psychomotor slowing and mild cognitive impairment with attention deficit and alterations in visuo-motor coordination and working memory (Amodio et al., 2005, and Bajaj et al., 2009) and severe motor and cognitive impairment (Felipo et al., 2012).

Distension of perivascular spaces was detected in cerebral cortex and striatum indicated mild cerebral edema in these areas. Previous studies showed that low grade cytotoxic cerebral edema was detected in $\mathrm{HE}$ and had been attributed to increased glutamine formation (Häussinger et al., 2000, and Häussinger, 2006). The magnetic resonance applied on patients with cirrhosis and $\mathrm{HE}$ confirmed presence of low-grade cerebral edema in cerebral cortex (lodi et al., 2004, and Kumar et al., 2008). In this respect, Cauli et al. (2013) found that weak role of cerebral edema in the neuropathological lesions in minimal or clinical chronic HE, due to low level of blood ammonia at this stage of chronicity.
Endothelial

capillary proliferation with hypertrophied endothelium were detected in many brain areas but were more severe in cerebral cortex this indicative for hypoxia exerted on this part of brain, these results confirmed by Butterworth (2009) who stated that cerebral blood flow in patients with cirrhosis is changed in a region-selective manner.

We can conclude that HE developed in chronic liver disease rat model is a pecular selective distribution pattern of neuropathy among brain areas, in which neuronal loss was severe in mid brain and pons compared with other brain areas. Astroglial and microglial reactions were prominent in most of brain regions indicating that the neuroinflammation play an important role in the mediation of HE.

\section{References}

Amodio P, Schiff S, Del Piccolo F, Mapelli D, Gatta A, Umiltà C (2005). Attention dysfunction in cirrhotic patients: an inquiry on the role of executive control, attention orienting and focusing. Metab Brain Dis.;20(2):115-27. doi: 10.1007/s11011-005-4149-3.

PMID: 15938130.

Bajaj JS, Saeian K, Schubert CM, Hafeezullah M, Franco J, Varma RR, Gibson DP, 
Hoffmann RG, Stravitz RT, Heuman DM, Sterling RK, Shiffman M, Topaz A, Boyett S, Bell D, Sanyal AJ (2009). Minimal hepatic encephalopathy is associated with motor vehicle crashes: the reality beyond the driving test. Hepatology.;50(4):1175-83. doi: 10.1002/hep.23128.

PMID: 19670416;

PMCID: PMC2757520.

Bancroft J. D (2013). Histochemical techniques. Butterworth-Heinemann.

Bismuth M, Funakoshi N, Cadranel JF, Blanc P. (2011). Hepatic encephalopathy from pathophysiology to therapeutic management.

GastroenterolHepatol; 23:822. [http://dx.doi.org/10.1097/MEG .0b013e32834 17567] [21099434].

Butterworth RF, Norenberg MD, Felipo V, Ferenci $P$, Albrecht J, Blei AT; Members of the ISHEN Commission on Experimental Models of $\mathrm{HE}$ (2009). Experimental models of hepatic encephalopathy: ISHEN guidelines. Liver Int. ;29(6):783-8. doi:

$10.1111 / \mathrm{j} .1478$

3231.2009.02034. x. PMID: 19638106.

Butterworth R. F. (2011). Neuroinflammation in acute liver failure: mechanisms and novel therapeutic targets,
Neurochem. Int. 59 (6) 830 836.

Cauli Marta Llansola Ana Agustí Regina Rodrigo Vicente Hernández-Rabaza, Tiago B. Rodrigues Pilar López-Larrubia Sebastián Cerdán Vicente Felipo.(2013). Cerebral oedema is not responsible for motor or cognitive deficits in rats with hepatic encephalopathy.

Cauli, M. Llansola, A. Agustí, R. Rodrigo, V. HernándezRabaza, T.B.Rodrigues, $P$. López-Larrubia， S. Cerdán, V. Felipo (2014). Cerebral oedema is notresponsible for motor or cognitive deficits in rats with hepaticencephalopathy, Liver Int. 34 (3), 379-387.

Dongmei

Qin, Yaru

Nie, and Zhiping Wen (2014) Protection of rats from thioacetamide-induced hepatic fibrosis by the extracts of a traditional

Uighur medicine Cichorium glandulosum. Iran J Basic Med Sci. ; 17(11): 879-885.

Felipo V, Ordoño JF, Urios A, El Mlili N, Giménez-Garzó C, Aguado C, González-Lopez O, Giner-Duran R, Serra MA, Wassel A, Rodrigo JM, Salazar J, Montoliu C (2012). Patients with minimal hepatic encephalopathy show impaired mismatch negativity correlating 
with reduced performance in attention tests. Hepatology;55(2):530-9. doi: 10.1002/hep.24704. PMID: 21953369.

Farjam M, Dehdab $\mathbf{P}$, Abbassnia F, Mehrabani D, Tanideh N, Pakbaz S, Imanieh MH. (2012). Thioacetamideinduced acute hepatic encephalopathy in rat: behavioral, biochemical and histological changes. Iran Red Crescent Med J. Mar;14(3):16470. Epub 2012 Mar 1. PMID: 22737573;

PMCID:

PMC3372030.

Häussinger D, Kircheis G, Fischer R, Schliess F, vom Dahl S (2000). Hepatic encephalopathy in chronic liver disease: a clinical manifestation of astrocyte swelling and lowgrade cerebral edema? J Hepatol.;32(6):1035-8. doi: 10.1016/s0168-8278(00)801105. PMID: 10898326.

Häussinger. D. (2006) Low grade cerebral edema and the pathogenesis of hepatic encephalopathy in cirrhosis. Hepatology; 43: 1187-90.

Kumar R, Gupta RK, Elderkin-Thompson V, (2008). Voxel based diffusion tensor magnetic resonance imaging evaluation of low-grade HE. J Magn Reson Imaging; 27: 1061-8.
Lodi R, Tonon C, Stracciari A, Weiger M, Camaggi V, Iotti S, Donati G, Guarino M, Bolondi L, Barbiroli B (2004). Diffusion MRI shows increased water apparent diffusion coefficient in the brains of cirrhotics. Neurology. 9;62(5):762-6. doi: 10.1212/01.wnl.0000113796.30 989.74. PMID: 15007127.

Montoliu C, Rodrigo R, Monfort P, Llansola M, Cauli O, Boix J, Elmlili N, Agusti A, Felipo V (2010). Cyclic GMP pathways in hepatic encephalopathy. Neurological and therapeutic implications. Metab Brain Dis. 2010 Mar;25(1):39-48. doi: 10.1007/s11011-010-9184-z.

PMID: 20195723.

Rodrigo. R., O. Cauli, U. Gomez-Pinedo, A. Agusti, V. Hernandez-Rabaza,

J.M.Garcia-Verdugo, V. Felipo (2010). Hyperammonemia induces neuroinflammation that contributes to cognitive impairment in rats with hepaticencephalopathy, Gastroenterology. 139 (2), 675684.

Romero-G_omez M, Boza F, Garcia-Valdecasas MS (2001). Subclinical hepatic encephalopathy predicts the development of overt hepatic encephalopathy. Am Gastroenterol; 96: 2718-23. 
Wallace; M.C, K Hamesch, M

Lunova, Y Kim, R

Weiskirchen, P Strnad, and SL

Friedman (2015). Laboratory

Animals.Vol. 49(S1) 21-29.

Weissenborn K, Bokemeyer

M, Ahl B, Fischer-Wasels D,
Giewekemeyer K, van den Hoff J, Köstler H, Berding G (2004). Functional imaging of the brain in patients with liver cirrhosis. Metab.

Dis.;19(3-4):269-80.

$$
\begin{aligned}
& \text { التقييم المقارن لتطور العلل العصبية في مناطق الدماغ المختلفة في نموذج }
\end{aligned}
$$

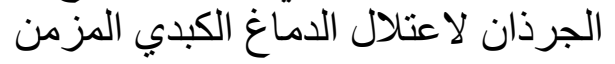

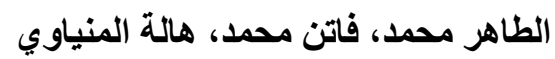

$$
\begin{aligned}
& \text { قسم الباثولوجيا، كلية الطب البيطري، مامعة هالية القاري } \\
& \text { الملخص العربى }
\end{aligned}
$$

صممت هذه التجربة لدراسة التأثير المزمن لتليف الكبد لأحداث الأعتلال العصبى لأنسجة

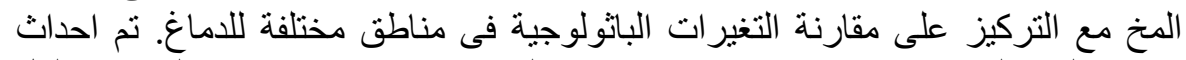

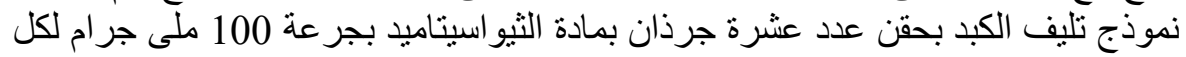

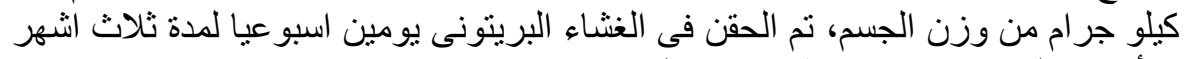

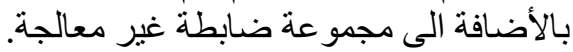

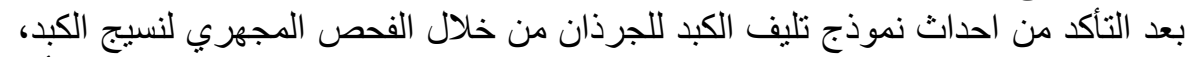

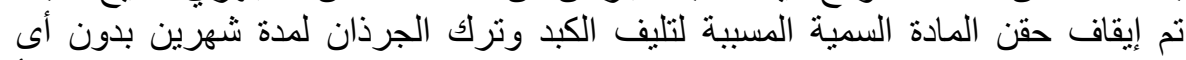

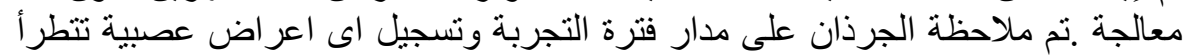
بعد انتهاء مدة التجربة تم القتل الرحيم للحيو انات واخذ عيننات لأجز اء الدماغ وتثبيتها فى الفى

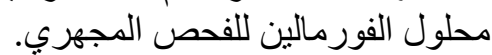

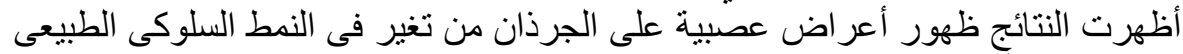

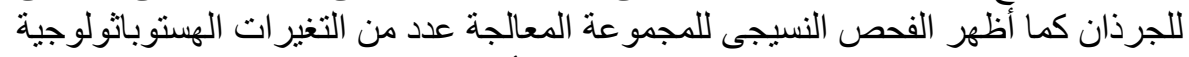

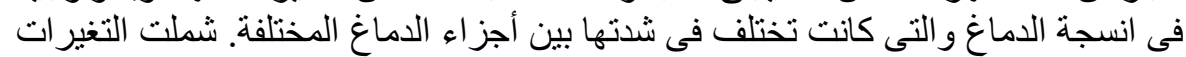

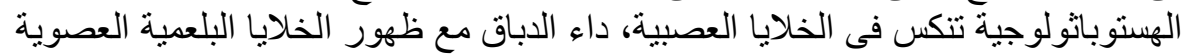

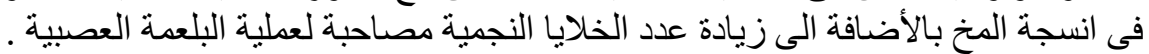

